O. Diekmann An invitation to structured (meta)population models 
CWI is the research institute of the Stichting Mathematisch Centrum, which was founded on February 11, 1946, as a non-profit institution aiming at the promotion of mathematics, computer science, and their applications. It is sponsored by the Dutch Government through the Netherlands organization for scientific research (NWO).

Copyright (C) Stichting Mathematisch Centrum, Amsterdam 


\title{
An Invitation to Structured (Meta)Population Models
}

\author{
O. Diekmann \\ $C W I$, \\ P.O. box 4079, 1009 AB Amsterdam, \\ The Netherlands \\ and \\ Institute of Theoretical Biology \\ Kaiserstraat 632311 GP Leiden \\ The Netherlands
}

\begin{abstract}
This paper presents a survey of some aspects of the formulation of mathematical models of physiologically (and spatially) structured populations. It consists of three parts. The first concentrates on the definition and the calculation of the basic reproduction ratio $R_{0}$, the intrinsic rate of natural increase $r$ and the asymptotic speed of propagation $c_{0}$ for a rather general class of models for population growth, or decline, in a constant environment. Part two introduces, by way of examples, the first order partial functional differential equations that arise when models for mechanisms at the individual level in variable environments are "lifted" to the population level. The idea that nonlinearity results from feedback through the environment is illustrated in the context of energy budget models for Daphnia populations. A numerical technique is briefly described. The third part is devoted to metapopulation models. It is argued that, and elaborated how, the formalism of the second part applies when we consider local populations as individuals characterized by colony size (or sizes, when several species are involved). Time scale arguments and special choices of model ingredients are used to derive tractable simplifications for which biologically relevant conclusions can be obtained.
\end{abstract}

AMS 1991 Mathematics Subject Classification: 92D25, 92D30

Key Words \& Phrases: basic reproduction ratio/reproductive rate $R_{0}$, asymptotic speed of propagation, energy budget models, Daphnia, first order partial functional differential equations, balance laws, escalator box car train, prey patches, predator-prey patches, time scale arguments.

Note: Partly supported by the U.S. Army Research Office through the Mathematical Sciences Institute of Cornell University.

\section{INTRODUCTION}

The contribution of an individual to population growth and interaction depends, as a rule, on various characteristics related to its physiology and its spatial position. Structured population models take this observation seriously and start the modelling process at the individual level ( $i$-level for short). First an $i$-state space $\Omega$ is specified and the movement of individuals through $\Omega$ is described in dependence on the state of the environment ( $E$-state). Likewise the way reproduction, death and influence on the environment depend on $i$-state and $E$-state are described. These ingredients at the $i$-level completely determine the deterministic formulation at the $p$-level ( $p$ for population): simple bookkeeping principles tell us how the $p$-equations should look. (Admittedly, however, the appearance of the $p$-equations depends somewhat on our choice of $p$-state space: either $L_{1}(\Omega)$ if we expect that the population distribution over $\Omega$ has a nice density, or $M(\Omega)$ if we expect that the distribution may contain measures concentrated on subsets of $\Omega$ ).

Here I shall restrict myself to models that, though having stochastic components at the $i$-level, in particular related to death and reproduction, are deterministic at the $p$-level. Concerning the biologymathematics interface (the "true" modelling) I shall be rather abstract, general and vague, but references are provided to published material where one can find concrete and detailed elaborations. My aim is to briefly introduce some techniques which are helpful in deriving biologically meaningful conclusions from an analysis of the infinite dimensional $p$-dynamical systems. These techniques range

Report AM-R9113

ISSN 0924-2953

CWI

P.O. Box 4079, 1009 AB Amsterdam, The Netherlands 
from positive operator theory and numerical analysis to formal time scale arguments.

Part I is concerned with the simplifications that arise, both in model formulation and in mathematical analysis, from the assumption that the environment is constant. A definition of the basic reproduction ratio $R_{0}$ is provided and computational aspects are discussed. Next the notion of "asymptotic speed of propagation" for homogeneous and isotropic infinite spatial domains is explained. References for the application of these ideas to the spread of infectious diseases and the invasion of new species are given.

Part II introduces energy budget models for Daphnia (and other organisms) as an example of structured models incorporating variable environments, where feedback through the environment leads to nonlinearity. A numerical method is briefly described.

Part III deals with metapopulation models, where local populations are considered as "individuals". It is shown that the formalism of structured population models is rich enough to incorporate many biological mechanisms, but that the resulting mathematical problem at the $p$-level is rather formidable. By model simplification, based on quasi steady-state assumptions, the neglect of delays and special choices of model ingredients, more tractable approximations are derived from which one can obtain qualitative conclusions.

\section{ACKNOWLEDGEMENT.}

The writing of this paper was initiated during a visit of three weeks at the Mathematical Sciences Institute, Cornell University. I like to thank Si Levin and Rick Durrett for their hospitality and stimulating discussions.

\section{PART I. INVASION IN A CONSTANT ENVIRONMENT}

\section{THE DEFINITION OF $R_{0}$.}

Considerr a population living under constant environmental conditions. The key question is: will the population grow or decline? In order to answer the question we can either count on a generation basis or with real time intervals as the basis for comparison (but of course the answer should not depend on our choice). We start to look from one generation to the next.

Let $B(\tau, \xi, \eta)$ denote the expected number of offspring with state $\xi$ at birth, produced per unit of time by an individual of age $\tau$ which was born with state $\eta$. We claim that $B$ gives all the information we need to settle the growth-or-decline question. To substantiate this claim we introduce the so-called next-generation operator $K$ :

$$
(K \phi)(\xi)=\int_{\Omega}\left(\int_{0}^{\infty} B(\tau, \xi, \eta) d \tau\right) \phi(\eta) d \eta
$$

The idea here is that $\phi$ describes both how many members a certain generation has (viz., $\int_{\Omega} \phi(\eta) d \eta$ ) and how these are distributed with respect to their state at birth. Then $K \phi$ does exactly the same for the next generation and that explains how $K$ arrived at its name. Our key question now translates into the following: what happens when we iterate $K$ ? It seems that in principle the answer could depend on the initial condition, the zero'th generation. In general (that is, under minor conditions on $B$ ), however, it does not. The reason is positivity. To serve as a meaningful model, both $\phi$ and $B$ should be nonnegative. Hence $K$ is a positive operator and, under the condition that the spectral radius $R_{0}$ of $K$, defined by

$$
R_{0}=\lim _{n \rightarrow \infty}\left\|K^{n}\right\|^{1 / n},
$$


is a strictly dominant eigenvalue of $K$ (i.e. the other spectral values of $K$ lie strictly inside the disk of radius $R_{0}$ in the complex plane), one finds that

$$
K^{n} \phi \sim c(\phi) R_{0}^{n} \phi_{d}, \quad n \rightarrow \infty,
$$

where $\phi_{d}$ is the eigenvector corresponding to $R_{0}$ and all dependence on the zero'th generation $\phi$ is through a scalar quantity $c$. We conclude that the answer to the key question is completely determined by $R_{0}$ : when $R_{0}>1$ the population will grow, when $R_{0}<1$ it will decline. In particular, invasion by a new species will be successful if and only if $R_{0}>1$.

Remarks: 1) Here we restrict ourselves to the deterministic setting at the population level. The stochastic formulation leads to multitype branching processes [12,18]. Although $R_{0}>1$ is then still a necessary condition for exponential growth, it is no longer a sufficient condition, since even in the supercritical case there exists a non-zero probability that extinction occurs.

2) Let $u(t, \xi)$ be the rate at which at time $t$ individuals are born with state $\xi$. Then

$$
u(t, \xi)=\int_{\Omega} \int_{0}^{\infty} B(\tau, \xi, \eta) u(t-\tau, \eta) d \tau d \eta
$$

If we substitute $u(t, \xi)=e^{\lambda t} \psi(\xi)$ we find that $\psi$ has to be an eigenvector corresponding to the eigenvalue one for the operator $K_{\lambda}$ defined by

$$
\left(K_{\lambda} \phi\right)(\xi)=\int_{\Omega}\left(\int_{0}^{\infty} B(\tau, \xi, \eta) e^{-\lambda \tau} d \tau\right) \phi(\eta) d \eta
$$

Monotonicity arguments imply that

$$
R_{0}>1 \Leftrightarrow r>0
$$

where $r$ is the unique real value of $\lambda$ for which $K_{\lambda}$ has dominant eigenvalue one. The population will grow, asymptotically for large time, at the rate $r$ (in other words, $r$ is the intrinsic rate of natural increase). Note that the time dependence in $B$ has no influence on $R_{0}$ but that it does influence the value of $r$ (in particular the value of $R_{0}-1$ does not by itself give any information about the magnitude of $r$ ).

\section{COMPUTATIONAL ASPECTS}

We discuss three special cases in which the determination of $R_{0}$ can be reduced to a manageable problem. [6,3]

Suppose that the probability distribution for state at birth is independent of the state at birth of the mother or, more precisely, assume that

$$
\int_{0}^{\infty} B(\tau, \xi, \eta) d \tau=a(\xi) b(\eta)
$$

Then $K$ has one-dimensional range spanned by $a$ (i.e. $K \phi$ is a multiple of $a$, no matter what $\phi$ we take) and consequently

$$
R_{0}=\int_{\Omega} b(\eta) a(\eta) d \eta
$$

So the independence allows us to obtain $R_{0}$ by averaging the life-time fertility $b$ with respect to the probability distribution $a$ of state at birth (assume, for the sake of the interpretation, that $\int_{\Omega} a(\eta) d \eta=1$ is used to normalize $a$ ).

Next consider the case 


$$
\int_{0}^{\infty} B(\tau, \xi, \eta) d \tau=a(\xi) b(\eta)+c(\eta) \delta(\eta-\xi)
$$

where $\delta$ is Dirac's "function." As an example of a biological situation where such an assumption might be an appropriate idealization, think of $i$-state as geographical position and two modes of seed dispersal: either seeds fall to the ground more ore less directly or they are blown into the air after which they can land essentially everywhere, irrespective of where they came from. Some straightforward algebraic manipulations lead to the conclusion that $R_{0}$ is the largest real root of the characteristic equation $f(\lambda)=1$, where by definition

$$
f(\lambda)=\int_{\Omega} \frac{b(\eta) a(\eta)}{\lambda-c(\eta)} d \eta
$$

Since $f$ is a monotone function of $\lambda$, finding its one point is rather easy.

Finally, consider the case where the state has a discrete component, i.e. $\xi=\left(i, \xi_{i}\right)$, and

$$
\int_{0}^{\infty} B\left(\tau,\left(i, \xi_{i}\right),\left(j, \xi_{j}\right)\right) d \tau=a_{i}\left(\xi_{i}\right) b_{i j}\left(\xi_{j}\right)
$$

The relation (3.5) is a conditional independence statement: conditional on the first component being $i$, the probability distribution for state-at-birth-component $\xi_{i}$ is fixed and independent of the state-atbirth of the parent. In the application to sexually transmitted diseases (where begetting offspring corresponds to infecting a susceptible) one may think of $i$ as distinguishing between men and women, while $\xi_{i}$ is an indicator of sexual activity. Define

$$
m_{i j}=\int_{\Omega_{j}} b_{i j}\left(\xi_{j}\right) a_{j}\left(\xi_{j}\right) d \xi_{j}
$$

then $R_{0}$ is the dominant eigenvalue of the positive matrix with entries $m_{i j}$. Thus we have reduced an infinite dimensional problem to a finite dimensional one, for which standard software is available.

\section{SUBMODELS FOR $B$}

In many situations $B$ has the representation

$$
B(\tau, \xi, \eta)=h(\tau) \int_{\Omega} c(\xi, \zeta) P(\tau, \zeta, \eta) d \zeta
$$

where $h$ is the expected intrinsic fecundity at age $\tau, c$ describes the distribution of the birth state $\xi$, given the current state $\zeta$ of the mother, while $P$ is the probability that the current state of an individual is $\zeta$, given that it started its life $\tau$ units of time ago with state $\eta$. Deterministic examples are

$$
P(\tau, \zeta, \eta)=\delta(\zeta-\eta)
$$

for a static $i$-state and

$$
P(\tau, \zeta, \eta)=\delta(\zeta-\eta-\tau)
$$

for the case where $i$-state equals age and $\Omega=[0, \infty)$. When $\Omega$ is discrete, say $\{1,2, \ldots, n\}$, and changes of $i$-state follow a Markov process with transition matrix $G$ we find

$$
P(\tau, i, j)=\left(e^{G \tau}\right)_{i j}
$$

and when $h$ is constant (and absorbed into $c$ ) we can easily perform the $\tau$-integration to obtain

$$
\int_{0}^{\infty} B(\tau, i, j) d \tau=\sum_{k=1}^{n} c(i, k)\left(-G^{-1}\right)_{k j}
$$

The specification of the coupling $c$ will depend heavily on the meaning of the $i$-state variable and 
nothing can be said in generality. The function $h$ may either be deduced from data, from an energy budget consideration (see Part II) or from some stochastic submodel. For instance, in the context of infectious diseases one considers compartmental models and probabilities per unit of time to go from one compartment to another, while infectivity depends on the compartment one is in. Such a situation is described by a $d$-state ( $d$ from disease) which takes values $1,2, \ldots, n$, a vector $b$ with $\sum_{i=1}^{n} b_{i}=1$, which gives the probability distribution for $d$ state at the moment immediately following infection, a $n \times n$-matrix $\Sigma$ of transition rates and a vector $q$ of infectivities. In terms of these ingredients one has

$$
h(\tau)=\left\langle q, e^{\Sigma \tau} b>\right.
$$

In epidemic models it is useful to factorize

$$
B(\tau, \xi, \eta)=\Lambda(S)(\xi) A(\tau, \xi, \eta)
$$

where $\Lambda(S)(\xi)$ describes how $B$ depends on the demographic steady state density of susceptibles $S$, as distributed with respect to $i$-state $\xi$. The strong form of the Law of Mass Action would yield

$$
\Lambda(S)(\xi)=S(\xi)
$$

but, for diseases transmitted during sexual intercourse or by biting mosquito's saturation effects lead to different functional forms for $\Lambda$. [11]

Epidemic models clearly illustrate the untenability of our assumption that environmental conditions stay constant: the density of susceptibles will actually decrease as the disease makes victims (at least when the disease leads to immunity or death and the epidemic time scale is much faster than the demographic time scale). The assumption is an idealization appropriate for the initial phase of population expansion or disease spread, when density dependence can still be ignored (that's why we speak about invasion.) Mathematically it amounts to linearization. Note, however, that the specification of a full nonlinear model may be a much more formidable task and that our idea to focus on $B$ directly has a certain economy of thought and parametrization. The main point of this section then, was to explicitly emphasize that $B$ may be composed from various building blocks, each of which may be related to a submodel for certain (biological) mechanisms.

When we have to iterate many times in (2.3) before the generations adopt the stable distribution as described by $\phi_{d}$, we may be at variance with the restriction to the initial phase of population expansion, as is implicit in the linearization giving constancy of environmental conditions. And indeed, the next section is devoted to a situation in which, though the sign of $R_{0}-1$ still decides whether or not invasion will be successful, $r$ does not give any information about the rate at which the invading organism will increase in numbers during the initial phase.

\section{THE ASYMPTOTIC SPEED OF PROPAGATION}

When we consider a fungal plant disease which spreads in a very large field by spore dispersal, we may take

$$
B(\tau, \xi, \eta)=h(\tau) V(|\xi-\eta|)
$$

for $\tau \geqslant 0$ and $\xi, \eta \in \mathbb{R}^{2}$. What happens when we introduce the disease at a localized spot? First there is a transient phase in which the details of the initial condition matter. Next there is an intermediate asymptotic phase in which the disease propagates in all directions in a wave like manner with a constant velocity $c_{0}$. When these waves reach the boundary a final phase begins in which the details of the geographic situation matter. In a truly infinite field the third phase does not occur and after the initial phase similarity solutions, which are plane waves travelling with velocity $c_{0}$, take over. [4]

The ingredients $h$ and $V$ determine the velocity $c_{0}$ as follows. For the linear birth-rate equation (2.4), with $B$ given by (5.1), the travelling plane wave "ansatz"

$$
u(t, \xi)=e^{\lambda\left(t+c \xi_{1}\right)}
$$


(where $\xi_{1}$ denotes the first component of $\xi$ ) leads to the equation

$$
L(c, \lambda)=1
$$

where by definition

$$
L(c, \lambda)=\int_{0}^{\infty} h(\tau) e^{-\lambda \tau} d \tau \int_{\mathbb{R}^{2}} V(|\eta|) e^{-\lambda c \eta_{1}} d \eta
$$

Provided $R_{0}=L(0,0)>1$ there exist solutions $\lambda>0$ whenever $c$ exceeds a critical number, which we call $c_{0}$. Hence $c_{0}$ is characterized by the simultaneous equations

$$
L\left(c_{0}, \lambda_{0}\right)=1, \frac{\partial L}{\partial \lambda}\left(c_{0}, \lambda_{0}\right)=0
$$

In his thesis [1] van den Bosch has worked through the following program:

- $\quad$ determine reasonable $h$ and $V$ from (quasi-) mechanistic submodels

- calculate $c_{0}$, either from (5.5) or by using approximation formulae

- compare with observed speeds for plant diseases and animal invasions

The paper [2] gives an account of the outcome of this program.

\section{OPERATIONALIZATION OF $R_{0}$ : SOME EXAMPLES}

In the last section it was indicated that one can use the general and somewhat abstract definition of the asymptotic speed $c_{0}$ as a tool in the quantitative analysis of concrete biological invasions. With $R_{0}$ one can do exactly the same thing. For a certain type of structure one can develop algorithms which compute $R_{0}$ from a specification of the various ingredients. The paper [13] gives an example in the context of veterinary epidemiology and in work in progress de Jong, Heesterbeek and Diekmann elaborate on this by allowing for a combination of the barn structure of a swine production farm and stage structure of the animal population, while exploiting the conditional independence conditions of section 3 to reduce the computer computations to finite dimensions. Ultimately this piece of bioindustrial mathematics should yield a valuable tool for the assessment of the efficacy of vaccination and other control measures.

When offspring is produced only in pairs which form a stable configuration for non-negligible periods of time, one cannot, in general, work with an age-representation to describe fecundity. This is particularly relevant for models for sexually transmitted diseases that take pair formation into account. But, as demonstrated in [5], our general methodology easily leads to the matrix which represents the next generation operator and which has $R_{0}$ as its dominant eigenvalue. In work in progress Dietz, Heesterbeek and Tudor analyse how, for a disease of given virulence, $R_{0}$ depends on the parameters of the pair formation and dissociation process.

\section{PART II: VARIABLE ENVIRONMENTS (AND NONLINEARITY BY FEEDBACK THROUGH THE ENVIRONMENT)}

\section{ENERGY BUDGET MODELS}

Let the individuals of a population be distinguished from one another by their size $x$. Assume that all are born with birth size $x_{b}$ and that they grow at a rate $g$ which depends on their size and on the prevailing food concentration $S$. Assume that individuals have a probability per unit of time $\mu=\mu(x, S)$ of dying and that they produce offspring at a rate $\lambda=\lambda(x, S)$ and consume food at a rate $\gamma=\gamma(x, S)$.

Let the density function $n$ describe the size and composition of the population, i.e.

$$
\int_{x_{1}}^{x_{2}} n(t, \xi) d \xi
$$

is the number of individuals, at time $t$, with size between $x_{1}$ and $x_{2}$. Then one can follow either 
Lagrange or Euler in the derivation of the bookkeeping equations (see [16] )

$$
\left\{\begin{array}{l}
\frac{\partial n}{\partial t}+\frac{\partial}{\partial x}(g n)=-\mu n \\
g\left(x_{b}, S\right) n\left(t, x_{b}\right)=\int_{x_{b}}^{\infty} \lambda(\xi, S) n(t, \xi) d \xi
\end{array}\right.
$$

these equations tell us how processes at the individual level express themselves at the population level. We can either think of $S$ as a given, time dependent variable (for instance when we deal with controlled experiments) or as a dynamic variable by itself (think of a field situation) which is governed by a differential equation such as

$$
\frac{d S}{d t}=\alpha S\left(1-\frac{S}{K}\right)-\int_{x_{b}}^{\infty} \gamma(\xi, S) n(\cdot, \xi) d \xi .
$$

In the latter case the combined problem (7.1) - (7.2) is nonlinear by feedback through the environment. Note, moreover, the non-local character (in particular in the boundary condition describing the appearance of new-borns.)

When the vital rates $\mu$ and $\lambda$ depend not only on size but also on age, equations (7.1) should be replaced by

$$
\left\{\begin{array}{l}
\frac{\partial n}{\partial t}+\frac{\partial n}{\partial a}+\frac{\partial}{\partial x}(g n)=-\mu n \\
n(t, 0, x)=\int_{0}^{\infty} \int_{0}^{\infty} \pi(y)(x) \lambda(\alpha, y, S) n(t, \alpha, y) d \alpha d y
\end{array}\right.
$$

where $\pi(y)(x)$ is the probability that offspring produced by a mother of size $y$ will be born with size $x$.

An energy budget model consists of the specification of $g, \lambda, \pi, \mu$ and $\gamma$ as functions of the $i$-state (either size $x$ alone or size $x$ and age $a$ ) and the environmental state (here the food concentration $S$ ) (see Kooijman [14]). Note that modelling is concerned with processes at the individual level and that straightforward mathematical bookkeeping suffices to "lift" the model to the population level.

The word "straightforward" in the last sentence above refers to the formulation of the model. To analyse the model and to arrive at biologically relevant conclusions is far less straightforward, unfortunately. Nevertheless some conclusions have been obtained for the age-size structured prey-predator model sketched above.

Apart from the familiar "paradox-of-enrichment" prey-predator cycles, the model can exhibit "demographic" cycles, in which time periods in which there are only a few large predators alternate with periods in which there are many small predators, in such a way that the total prey consumption is nearly constant. This dynamic regime manifests itself for small values of prey carrying capacity $K$ and small values of the predator death rate $\mu$. It does explain experimental observations on Daphnia: Murdoch and McCauley [15] found that sometimes numbers of Daphnia oscillate wildly while the algae concentration remains nearly constant.

The dynamic behaviour described above was found by de Roos and co-workers [20,18,21]. A key tool in the analysis is a numerical technique, called the escalator box car train, to integrate first order partial differential equations like (7.1) and (7.3), involving non-local boundary conditions. The technique is based on an approximation of the density function $n$ by cohorts (or, in more mathematical jargon, by concentrated measures) which reduces the partial differential equation to a finite system of 
ordinary differential equations for which numerical methods are readily available. Existing cohorts change in number due to death

$$
\frac{d n_{i}}{d t}=-\mu\left(\xi_{i}, S\right) n_{i}
$$

and in mean size $\xi$ due to growth

$$
\frac{d \xi_{i}}{d t}=g\left(\xi_{i}, S\right)
$$

(and in mean age, when age is another $i$-state variable). Here $i$ refers to the cohort number, a purely administrative index which we may adjust at regular time intervals, just as in the discretized age Lesley matrix model, to keep the range of $i$-values within bounds. The only non-trivial element of the technique is the derivation of ordinary differential equations for "cohorts in creation", i.e. for cohorts which are gradually formed from newborns (there is just one such cohort in the case of (7.1), but there may be several of them needed along the boundary $a=0$ in the case of (7.3)). A detailed exposition of this aspect is presented in [21]. I conclude this part by emphasizing that, in my opinion, an important attractive feature of this technique is that the resulting system of ordinary differential equations has a clear interpretation as a biological model itself! In fact in [21] the equations are derived directly, and not as a numerical approximation to a partial differential equation.

Finally, I refer to work by Kooijman [14] on models which take energy reserves into account.

\section{PART III: MeTAPOPULATION MODELS}

\section{LOCAL POPULATIONS CONSIDERED AS INDIVIDUALS}

When we want to describe an ensemble of local patches, we may conceive of a local population as an individual, characterized by its local population size. When these patches are arenas for interaction, there are several sizes to take into account, and the $i$-state space becomes higher-than-one dimensional. In the present paragraph we concentrate on the interaction of phytophages (spider mites) with their food source (plants) and their natural enemies (predator mites). So a local colony is characterized by

1. the amount of food (leaf area) $z$

2. the number of phytophages/prey $x$

3. the number of predators $y$

Our basic assumptions are that:

- new prey colonies are founded by prey emigrating from existing colonies

- prey colonies come to an end when the local food source is over-exploited

- predator invasion in a prey colony will, after a while, lead to extermination of the prey followed by dispersion of the remaining predators.

So what we need are submodels for the local tritrophic interaction:

- food production

- prey increase/food decline

- predator increase/prey decline

and for the non-local process of dispersion:

- tendency to emigrate

- dynamics of "aireal plankton" (starvation, search)

- foundation of prey colonies

- predator invasion in prey colonies. 
Here one should note that geographical structure is not explicitly taken into account (no "distance"), but only implicitly through the fact that we acknowledge the existence of local colonies. But what exactly qualifies to be called a local colony? In the real world we see the same phenomena at different spatial (and temporal) scales (leaves, twigs, branches, shrubs, ...). Once we decide about the scale on which we want to concentrate we can be more precise about the definition of a local colony. (The structure of the equations introduced below does not depend on our choice, but of course the numerical values of the parameters do). The key feature is that movement within a patch should be easy and frequent relative to movement from one patch to another.

In the next section we shall formulate mathematical models that are in the spirit of the above considerations, but still relatively simple. For instance, we shall assume that all patches are equal as far as food is concerned (but see Hanski and Gyllenberg [10,9]) and we shall not model the food dynamics. Neither shall we take into account that parameters do depend on temperature and other meteorological variables.

\section{Deriving the Population Balance LaWs}

Let $x$ denote prey colony size and let the density function $n$ be such that

$$
\int_{x_{1}}^{x_{2}} n(t, \xi) d \xi
$$

is the number of prey patches at time $t$ of a size between $x_{1}$ and $x_{2}$. let $v(x)$ be the rate of growth of $x$. Concerning the rate at which predators invade a prey colony of size $x$ we assume that it factorizes as a product of a vulnerability index $\eta(x)$ and the prevailing density of predator aireal plankton $Q(t)$. Then the time evolution of the population state $n$ is described by the pde

$$
\frac{\partial}{\partial t} n(t, x)+\frac{\partial}{\partial x}(v(x) n(t, x))=-\eta(x) Q(t) n(t, x)
$$

together with the boundary condition

$$
v(1) n(t, 1)=\zeta n_{0}(t) P(t)
$$

where $n_{0}$ is the number of suitable "free" patches, $P$ is the density of prey aireal plankton and $\zeta$ is a "reaction" constant. There is a strange but deliberate inconsistency here. Whereas prey colony growth is described by a differential equation for a continuous variable $x$, the founding of the colony is described by a discrete change $x=0 \rightarrow x=1$. The argument here is that after a very short initial phase population growth is deterministic to a good approximation, with further immigration negligible relative to reproduction.

Let $y$ denote the size of the predator population in a certain patch and let the density function $m$ be such that

$$
\int_{x_{1}}^{x_{2}} \int_{y_{1}}^{y_{2}} m(t, x, y) d y d x
$$

is the number of predator patches at time $t$ with prey population size between $x_{1}$ and $x_{2}$ and predator population size between $y_{1}$ and $y_{2}$. The local predator-prey interaction is assumed to be described by the system of ode's

$$
\begin{aligned}
& \frac{d x}{d t}=g(x, y) \\
& \frac{d y}{d t}=h(x, y)
\end{aligned}
$$

where consistency requires that $v(x)=g(x, 0)$. As a consequence we find at the population level the 
balance law

$$
\frac{\partial}{\partial t} m(t, x, y)+\frac{\partial}{\partial x}(g(x, y) m(t, x, y))+\frac{\partial}{\partial y}(h(x, y) m(t, x, y))=0
$$

with the boundary condition

$$
h(x, 1) m(t, x, 1)=\eta(x) Q(t) n(t, x)
$$

describing the transmutation of prey patches into predator patches upon invasion.

We assume that the ode system $(9.3)$ is such that orbits starting at $(x, 1)$ reach the boundary $x=0$ after finite time or, in biological words, that predators do exterminate the local prey population. So the ode system determines somewhat implicitly both the interaction period and the predator yield at the end of that period. Note, finally, that preys may emigrate from predator invaded patches before extermination is a fact.

In the present context the "environment" consists of the aireal plankton $P$ and $Q$. We assume that dispersing prey and predators die with probability per unit of time, respectively, $\mu$ and $\nu$. The production of prey plankton is described by the emigration rate $\pi(x, y)$ and we obtain as the differential equation for $P$ :

$$
\frac{d P}{d t}(t)=\int_{1}^{\infty} \pi(x, 0) n(t, x) d x+\int_{1}^{\infty} \int_{0}^{\infty} \pi(x, y) m(t, x, y) d y d x-\mu P(t)
$$

For predator plankton, on the other hand, production is related to the massive dispersal from patches in which the prey population is exterminated. So we have to multiply the flux through the boundary $x=0$ by the yield $y$ and sum over $y$. This leads to

$$
\frac{d Q}{d t}(t)=-\int_{0}^{\infty} y g(0, y) m(t, 0, y) d y-\nu Q(t)
$$

Along the same lines as followed above one may introduce the food (leaf area) in a given patch as another state variable $z$ and work with densities $n_{0}(t, z), n(t, x, z)$ and $m(t, x, y, z)$. In doing so one increases not only the generality but also the complexity of the model considerably.

Our ideal now is to understand the global dynamical behaviour of the nonlinear infinite dimensional system described by (9.1), (9.2), (9.4)- (9.7) and how this behaviour depends on the various ingredients (submodels and parameters). Unfortunately this is an impossible task. The next section describes some techniques which allow us to achieve less ambitious goals. The main message of the present section is simply that the formalism of structured population models is rich enough to incorporate a great number of biological mechanisms.

\section{SOME LIMITING CASES}

The processes of dispersal, prey colony growth and local prey-predator interaction all have their characteristic time scale and these need not be the same. If some of these scales are widely different we may use either quasi-steady-state approximation or neglect some of the delays between cause and effect to obtain less complicated models. Moreover, even if these time scales are actually not very different one may still adopt the sound mathematical strategy of studying limiting special cases first before tackling the full problem. Most of the time insight obtained from special simplified cases is of much help in the analysis of the general case. Last but not least any qualitative understanding of close relatives of complicated models can be a key factor in the design and sensitivity analysis of computer experiments. 


\subsection{INSTANTANEOUS PREY EXTERMINATION AFTER PREDATOR INVASION}

When the time between predator invasion and prey extermination is negligible compared to the average time of dispersal and prey colony growth, we may forget about (9.4) and (9.5), drop the term involving $m$ in (9.7) and describe the dynamics of $Q$ by

$$
\frac{d Q}{d t}(t)=\delta Q(t) \int_{1}^{\infty} x \eta(x) n(t, x) d x-\nu Q
$$

where $\delta$ is the prey-to-predator conversion factor. As a further simplification we may consider the special case where $n_{0}$ is constant. The point of this is that one obtains a closed system of ode's for $O, P$ and $Q$ where, by definition,

$$
O(t)=\int_{1}^{\infty} x n(t, x) d x
$$

A straightforward analysis (see [7]) shows that the ode system has an asymptotically stable steady state. Comparison with the neutral stability of the Volterra-Lotka system then leads to the conclusion that

a prey dispersal phase of non-negligible duration has $\bar{a}$ stabilizing effect on the global prey-predator interaction.

The prey dispersal phase acts as a temporary refuge. In contrast [7]

a predator dispersal phase acts as a destabilizing delay

So if we treat the free patches as a dynamic variable things become more complicated since the herbivores consume (i.e. act as predator towards) plants but are prey for their predators. In [23] it is found that

a dispersal phase for the middle level in a tritrophic system has a destabilizing effect.

\subsection{CONSTANT INTERACTION TIME, PREDATOR YIELD, VULNERABILITY AND PREY DISPERSAL RATE}

The prey-predator interaction time (i.e. the time between predator invasion and prey extermination) as well as the predator yield at the end of the interaction depend on the prey colony size at the time of predator invasion. The precise form of this dependence is determined by the solutions of the ode system (9.3). The pde (9.1) adds to this no more and no less than the bookkeeping of the number of patches. So if we make alternative assumptions concerning the interaction time and the predator yield we may forget about (9.3) and (9.1). In this subsection we shall assume that both are constant, i.e. independent of the prey colony size at the time of predator invasion.

Under this assumption prey colony size is still relevant since it determines the vulnerability $\eta$, the prey dispersal rate $\pi$ and, finally, the probability that the patch will crash. But if we assume that all these parameters are, in fact, independent of prey colony size, we may dispose of $n$ and work with the total number of prey patches

$$
N(t)=\int_{1}^{\infty} n(t, x) d x
$$

instead. One then arrives at a system of differential delay equations (which can still be further simplified by adopting quasi-steady-state approximations for $P$ and $Q$ ). Standard steady state stability analysis yields the following conclusions:

[22,23]

and

postponement of predator dispersion to the end of the interaction period is a destabilizing mechanism. 


\subsection{INSTANTANEOUS HOST PLANT DESTRUCTION, POSSIBLY DEFEATED BY PREDATOR INVASION}

Whenever the prey exhaust their host plant very quickly we may employ a somewhat more sophisticated time scale argument. In the absence of predators the founding of a prey colony leads instantaneously to the production of new searching prey. When predators are around they may invade and then the instantaneous yield consists of predators rather than prey. The probability of predator invasion as well as the predator yield after invasion depend on predator aireal plankton density $Q$ in a manner to be derived from the limiting procedure (see [7] Appendix). The end result is a system of three ode's for $n_{0}, P$ and $Q$. Remarkably, the system exhibits bistability in certain regions of parameter space [8], whence the conclusion:

for successful biological control one possibly needs to introduce many predators.

\section{REFERENCES}

[1] F. VAN DEN BosCH, The velocity of spatial population expansion, thesis, Leiden University, 1990.

[2] F. Van den Bosch, J.A.J. MeTZ, O. Diekmann The velocity of spatial population expansion, J. Math. Biol. 28 (1990) 529-556.

[3] O. Diekmann, Modelling infectious diseases in structured populations, Proceedings of the 11th Dundee Conference on the Theory of Ordinary and Partial Differential Equations, in press.

[4] O. Diekmann, Dynamics in Bio-Mathematical Perspective, In: Mathematics and Computer Science II (M. Hazewinkel, J.K. Lenstra, L.G.L.T. Meertens, eds.) CWI Monograph 4, NorthHolland (1986) 23-50

[5] O. Diekmann, K. Dietz, J.A.P. HeEsterbeek The basic reproduction ratio $R_{0}$ for sexually transmitted diseases, part I: Theoretical considerations, preprint, to appear in Math. Biosc.

[6] O. Diekmann, J.A.P. Heesterbeek, J.A.J. Metz On the definition and the computation of the basic reproduction ratio $R_{0}$ in models for infectious diseases in heterogeneous populations, J. Math. Biol. 28 (1990) 365-382.

[7] O. Diekmann, J.A.J. Metz, M.W. Sabelis Mathematical models of predator-prey-plant interaction in a patchy environment, Experimental and Applied Acarology 5 (1988) 319-342.

[8] O. Diekmann, J.A.J. Metz, M.W. SABelis Reflections and calculations on a prey-predator-patch problem, Acta Applicandae Mathematicae, 14 (1989) 23-35.

[9] M. Gyllenberg, I. Hanski, Single-species metapopulation dynamics: a structured model, Theor. Pop. Biol., in press.

[10] I. HANSKI, M. Gyllenberg, Two general metapopulation models and the core-satellite species hypothesis, preprint Lulea University.

[11] J.A.P. HeESTERBEeK, J.A.J MeTz, The saturating contact rate in epidemic models, preprint.

[12] P. JAGers, O. NeRman, The growth and composition of branching populations, Adv.Appl. Prob. 16 (1984) 221-259.

[13] M.C.M. DE Jong, O. Diekmann, A method to calculate - for computer-simulated infections - the threshold value, $R_{0}$, that predicts whether or not the infection will spread, preprint, to appear in Prev.Vet.Med.

[14] S.A.L.M. Kooljman, Population dynamics on basis of budgets, pp. 453-473 in reference [16].

[15] E.McCauley, W.W. Murdoch, Cyclic and stable populations: Plankton as a paradigm, Amer.Nat. 129 (1987) 97-121.

[16] J.A.J. Metz, O. Diekmann (eds) The Dynamics of Physiologically Populations, Lecture Notes in Biomathematics 68, Springer-Verlag, 1986.

[17] J.A.J. MeTz, O. Diekmann, Exact finite dimensional representations of models for physiologically structured populations. I. the abstract foundations of linear chain trickery. In: J.A. Goldstein, F. Kappel, W. Schappacher (eds.), Differential Equations with Applications in Biology, Physics and Engineering, Lecture Notes in Pure and Applied Mathematics 133 (1991) 269-289 (Marcel Dekker). 
[18] J.A.J. MeTZ, A.M. De Roos, F. VAN DEN BosCh, Population models incorporating physiological structure: a quick survey of the basic concepts and an application to size-structured population dynamics in waterfleas. In: B. Ebenman, L. Persson (eds.) Size-structured populations: ecology and evolution, Springer, Berlin (1988) 106-124.

[19] C.J. ModE, Multitype branching processes, theory and applications., Elsevier, New York, 1971.

[20] A.M. De Roos, J.A.J. MetZ, E. Evers, A. LeIPOLDT, A size-dependent predator-prey interaction: Who pursues whom? J. Math.Biol. 28 (1990) 609-643.

[21] A.M. DE Roos, O. Diekmann, J.A.J. MeTz, Studying the dynamics of structured population models: a versatile technique and its application to Daphnia, preprint, to appear in Amer.Nat.

[22] M.W. Sabelis, O. Diekmann, Overall population stability despite local extinction: The stabilizing influence of prey dispersal from predator invaded patches, Theor.Pop.Biol. 34 (1988) 169-176.

[23] M.W. SABELIS, O. Diekmann, V.A.A. JanSEn, Metapopulation persistence despite local extinction: predator-prey patch models of the Lotka-Volterra type, Biol. J. Linnean Soc. 42 (1991) 267-283. 
